\title{
PROJECTIVE MODULES OVER \\ LAURENT POLYNOMIAL RINGS
}

BY

RICHARD G. SWAN

\begin{abstract}
Quillen's solution of Serre's problem is extended to Laurent polynomial rings. An example is given of a $A\left[T, T^{-1}\right]$-module $P$ which is not extended even though $A$ is regular and $P_{\mathrm{m}}$ is extended for all maximal ideals $\mathfrak{m}$ of $A$.
\end{abstract}

The object of this note is to present several comments and examples related to some problems suggested by Quillen's recent solution of Serre's problem [7]. It is an immediate consequence of Quillen's work that the analogue of Serre's problem for Laurent polynomials is also true. In other words, if $G$ is a free abelian group and $k$ is a field or principal ideal domain, then all finitely generated projective $k G$-modules are free. This follows just as in [7, Theorem 4] once we observe that the following analogue of [7, Theorem 3] is true: If $P$ is a finitely generated projective $A\left[x, x^{-1}\right]$-module and if $A(x) \otimes_{A\left[x, x^{-1}\right]} P$ and $A\left(x^{-1}\right) \otimes_{A\left[x, x^{-1}\right]} P$ are both free, then $P$ is free. The proof is the same as that of [7, Theorem 3]. We note that $P$ extends over each half of the projective line over $\boldsymbol{A}$ and apply [7, Theorem 2].

This observation suggests extending the conjecture mentioned in [7] to the case of Laurent polynomial rings $A\left[x, x^{-1}\right]$. By combining Quillen's methods with those of Suslin and Vaserstein one can show that the conjecture is true for projective modules of sufficiently high rank. In \$2, I will give an example to show that some such restriction is really needed for the case of Laurent rings. The example also gives a negative answer to the question raised at the end of [7]. Furthermore, it shows that the analogue of [7, Theorem 1] for Laurent rings does not hold.

The conjecture of [7] would follow easily if one could extend Theorem 1 of [7] to the case of schemes. However, I will show in $\$ 3$ that this extension is also false.

1. Laurent rings. The question is to decide when a finitely generated projective module $P$ over $A\left[x, x^{-1}\right]$ is extended i.e., has the form $P \approx$ $A\left[x, x^{-1}\right] \otimes_{A} Q$. A partial answer to this is given by the following result

Received by the editors June 16, 1976.

AMS (MOS) subject classifications (1970). Primary 13C10, 16A50; Secondary 13B25, 55F25.

Key words and phrases. Projective modules, Laurent polynomial rings, Serre's problem.

- American Mathematical Society 1978 
which includes the main results of $[10]\left({ }^{1}\right)$.

THEOREM 1.1. Let $R$ be a commutative noetherian ring and let $P$ be a finitely generated projective module over

$$
A=R\left[x_{1}, x_{1}^{-1}, \ldots, x_{n}, x_{n}^{-1}, y_{1}, \ldots, y_{m}\right]
$$

where the $x_{i}$ and $y_{j}$ are indeterminates. Assume

(1) $[P] \in \operatorname{im}\left[K_{0}(R) \rightarrow K_{0}(A)\right]$,

(2) $r k P>$ Krull $\operatorname{dim} R$.

Then $P$ is extended from $R$ i.e., $P \approx A \otimes_{R} Q$.

We can give an alternative formulation of this result as a cancellation theorem.

Corollary 1.2. Let $R, A$, and $P$ be as in Theorem 1.1. Assume conditions (1) and (2) are satisfied. If $P^{\prime}$ is a projective $A$-module stably isomorphic to $P$, then $P^{\prime} \approx P$.

It is easy to see that this is equivalent to Theorem 1.1. Retract $A$ onto $R$ by sending $x_{i}$ to 1 and $y_{i}$ to 0 . Let $Q=R \otimes_{A} P$ and $Q^{\prime}=R \otimes_{A} P^{\prime}$. Since $P^{\prime}$ is stably isomorphic to $P$ it also satisfies (1) and (2) so $P \approx A \otimes_{R} Q$ and $P^{\prime} \approx A \otimes_{R} Q^{\prime}$. But $Q$ and $Q^{\prime}$ are stably isomorphic and Bass' cancellation theorem [1, Chapter IV, Corollary 3.5] applies because of (2). Therefore $Q^{\prime} \approx Q$ and so $P^{\prime} \approx P$. Conversely, if Corollary 1.2 holds and $P$ satisfies (1) and (2) then $P$ is stably isomorphic to $A \otimes_{R} Q$ by (1) and so $P \approx A \otimes_{R} Q$.

We now turn to the proof of Theorem 1.1. Consider first the case $n=0$ in which $A=R\left[y_{1}, \ldots, y_{m}\right]$ is a polynomial ring. This case was also done independently by Murthy. Conditions (1) and (2) are clearly preserved by localizing. It will suffice to show that $P_{\mathrm{m}}$ over $R_{\mathrm{m}}\left[y_{1}, \ldots, y_{m}\right]$ is extended for each maximal ideal $\mathfrak{m}$ of $R$ since the proof of [7, Theorem 1] clearly applies to polynomials in several variables. In fact, it applies to all graded $R$-algebras of the form $A=A_{0}+A_{1}+\ldots$ with $A_{0}=R$. Therefore we can assume $R$ is local. In this case, (1) simply says that $P$ is stably free while the conclusion says that $P$ is free. It will therefore suffice to treat the case where $P$ is presented by a unimodular row $\left(f_{0}, f_{1}, \ldots, f_{r}\right)$. By $[3, \S 4]$ or $[10$, Corollary 4.4] we can change variables and perform elementary transformations to make $f_{0}$ monic in $y_{m}$. Now $P_{f_{0}}$ is clearly free so [7, Theorem 3] applied to $R\left[y_{1}, \ldots, y_{m-1}\right]$ and $T=y_{m}$ shows that $P$ is free.

(') After this paper was written, Bass showed me a letter from Suslin in which he gives another proof of Serre's conjecture which was obtained independently of Quillen's work and at about the same time. His proof is completely different from Quillen's and uses ideas related to [11]. Suslin also states that his method yields Theorem 1.1 and Corollary 1.2 when $R$ is regular. Thus the only thing new in the present formulation of Theorem 1.1 and Corollary 1.2 is the inclusion of the nonregular case. 
For the case $n>0$ we need a slightly more general form of the argument of Murthy $[2, \S 4.2$, p. 25] which was used in [7, Theorem 3]. I will say that a finitely generated projective $R\left[x, x^{-1}\right]$-module $P$ extends to $R[x]$ if $P \approx$ $R\left[x, x^{-1}\right] \otimes_{R[x]} Q$ where $Q$ is a finitely generated projective $R[x]$-module.

LEMma 1.3. Let $P$ be a finitely generated projective $R\left[x, x^{-1}\right]$-module. Let $f \in R[x]$ be monic. If $P_{f}$ is extended from $R$, then $P$ extends to $R\left[x^{-1}\right]$.

Proof. Let $g\left(x^{-1}\right)=x^{-n} f(x)$ where $n=\operatorname{deg} f$. We can write $P_{f}=$ $R\left[x, x^{-1}\right]_{f} \otimes_{R\left[x^{-1}\right]} Q$ since $P_{f}$ is even extended from $R$. Regard $P$ as a vector bundle on Spec $R\left[x, x^{-1}\right]=\left\{x^{-1} \neq 0\right\}$ in Spec $R\left[x^{-1}\right]$. Take the bundle defined by $Q$, restrict it to $\{g \neq 0\}$ and glue the bundles together using the isomorphism over $\left\{x^{-1} \neq 0\right\} \cap\{g \neq 0\}=\operatorname{Spec} R\left[x, x^{-1}\right]$. These two open sets cover Spec $R\left[x^{-1}\right]$.

We can now finish the proof of Theorem 1.1. By induction on $n$ we can assume that Theorem 1.1 (and hence Corollary 1.2) holds for

$$
B=R\left(x_{1}\right)\left[x_{2}, x_{2}^{-1}, \ldots, x_{n}, x_{n}^{-1}, y_{1}, \ldots, y_{m}\right] .
$$

Retract $A$ onto $R$ as above and let $Q=R \otimes_{A} P$ so that $P$ and $A \otimes_{R} Q$ are stably isomorphic. Therefore $B \otimes_{A} P$ and $B \otimes_{R} Q$ are stably isomorphic so $B \otimes_{A} P \approx B \otimes_{R} Q$ by Corollary 1.2. Note that $\operatorname{dim} R\left(x_{1}\right)<\operatorname{dim} R$ by the remark used to prove [7, Lemma 2]. It follows that we can find a monic polynomial $f \in R\left[x_{1}\right]$ such that $P_{f} \approx A_{f} \otimes_{R} Q$. Let $C=R\left[x_{2}, x_{2}^{-1}, \ldots, x_{n}\right.$, $\left.x_{n}^{-1}, y_{1}, \ldots, y_{m}\right]$ so that $A=C\left[x, x^{-1}\right]$. By Lemma 1.3, $P$ extends to $P^{\prime}$ over $C\left[x^{-1}\right]$. Consider the maps

$$
K_{0}(R) \rightarrow K_{0}\left(C\left[x^{-1}\right]\right) \stackrel{i}{\rightarrow} K_{0}\left(C\left[x, x^{-1}\right]\right) .
$$

We know that $[Q] \in K_{0}(R)$ and $\left[P^{\prime}\right] \in K_{0}\left(C\left[x^{-1}\right]\right)$ have the same image $[P]$ in $K_{0}\left(C\left[x, x^{-1}\right]\right)=K_{0}(A)$. But $i$ is injective by [1, Chapter XII, Corollary 7.6] or, more simply, by [7, Theorem 3], so [ $\left.P^{\prime}\right]$ is the image of $[Q]$ in $K_{0}\left(C\left[x^{-1}\right]\right)$. This shows that the hypotheses of Theorem 1.1 are satisfied by $P^{\prime}$ over

$$
C\left[x^{-1}\right]=R\left[x_{2}, x_{2}^{-1}, \ldots, x_{n}, x_{n}^{-1}, y_{1}, \ldots, y_{m}, x_{1}^{-1}\right] .
$$

By induction on $n$ we conclude that $P^{\prime}$ (and hence $P$ ) is extended from $R$.

COROLLARY 1.4. If $R$ is noetherian of Krull dimension $<1$, then every finitely generated projective module $P$ over

$$
A=R\left[x_{1}, x_{1}^{-1}, \ldots, x_{n}, x_{n}^{-1}, y_{1}, \ldots, y_{m}\right]
$$

with $[P] \in \operatorname{im}\left[K_{0}(R) \rightarrow K_{0}(A)\right]$ is extended from $R$.

Proof. Since rk $P$ is locally constant on Spec $R$ we can write $R=R_{1}$ $\times \cdots \times R_{s}$ with $R_{i} \otimes_{R} P$ of constant rank (cf. [11, p. 428]). Therefore we 
can assume that $P$ has constant rank. If rk $P>2$, use Theorem 1.1. If rk $P=1$ use [1, Chapter IX, §3].

A natural question is whether one can drop the hypothesis (2) in Theorem 1.1. The example given in $\$ 2$ shows that this is not so if $n \geqslant 1$. However, the case $n=0$ remains open. There are several equivalent formulations of this.

(A) Let $R$ be a commutative ring and let $P$ be a finitely generated projective $R[x]$-module with $[P] \in \operatorname{im}\left[K_{0}(R) \rightarrow K_{0}(R[x])\right]$. Is $P$ extended?

(B) If $P$ is stably free over $R[x]$, is $P$ extended?

(C) Let $\left(f_{0}(x), \ldots, f_{n}(x)\right)$ be a unimodular row over $R[x]$ with $f_{0}(0)=1$, $f_{1}(0)=\cdots=f_{n}(0)=0$. Can this row be completed to an invertible matrix?

The equivalence of (A), (B), and (C) follows from [7, Theorem 1] which shows that it is sufficient to consider these questions when $R$ is local. In this case the equivalence is obvious.

Note that the hypothesis of (A) is equivalent to: For each maximal ideal $\mathrm{m}$ of $R$, the $R_{\mathrm{m}}[x]$-module $P_{\mathrm{m}}$ is stably free. This results from the following lemma which is an immediate consequence of [7, Theorem 1]. We let $N K_{0}(A)=\operatorname{ker}\left[K_{0}(A[x]) \rightarrow K_{0}(A)\right]$ as in [1, Chapter XII].

LEMMA 1.5. If $A$ is any commutative ring, then $N K_{0}(A) \rightarrow \Pi N K_{0}\left(A_{m}\right)$ is injective.

The product is over all maximal ideals of $A$.

Proor. Let $\alpha \in N K_{0}(A)$ map to 0 in all $N K_{0}\left(A_{\mathrm{m}}\right)$. Write $\alpha=[P]-[F]$ where $P$ and $F$ are finitely generated projective modules over $A[x]$ with $F$ free. For any maximal ideal $\mathrm{m}$ of $A$ we have $\left[P_{\mathfrak{m}}\right]-\left[F_{\mathfrak{m}}\right]=0$ so $\left(P \oplus F^{\prime}\right)_{\mathfrak{m}} \approx$ $\left(F \oplus F^{\prime}\right)_{\mathrm{m}}$ for some finitely generated free module $F^{\prime}$. Therefore we can find $s \in A-\mathfrak{m}$ with $\left(P \oplus F^{\prime}\right)_{s} \approx\left(F \oplus F^{\prime}\right)_{s}$. The set of $s$ so obtained (for all $\mathrm{m}$ ) generates the unit ideal so we can find a finite number of such $s_{i}$ with

$$
\sum A s_{i}=A \text { and }\left(P \oplus F_{i}^{\prime}\right)_{s_{i}} \approx\left(F \oplus F_{i}^{\prime}\right)_{s_{i}}
$$

Let $F^{\prime \prime}$ be free of rank $\geqslant$ rk $F_{i}^{\prime}$ for all $i$. Then $\left(P \oplus F^{\prime \prime}\right)_{s_{i}} \approx\left(F \oplus F^{\prime \prime}\right)_{s_{i}}$ so $P \oplus F^{\prime \prime} \approx F \oplus F^{\prime \prime}$ by [7, Theorem 1].

In [10, Theorem 8.1], I showed that a very special case of question (C) is true, namely the case where $n=2, \frac{1}{2} \in R$, and all $f_{i}$ have degree $<1$. If we assume the theorem of Suslin mentioned in $[10, \S 8]$, this can be generalized a bit.

TheOREM 1.6. Let $A$ be a commutative ring and let $\left(f_{0}, \ldots, f_{n}\right)$ be a unimodular row over $A[x]$ which defines a projective module $P$. Assume

(1) $f_{i}=a_{i}+b_{i} x$ with $a_{i}, b_{i} \in A$,

(2) $P / x P$ is free,

(3) $1 / n ! \in A$.

Then $P$ is free. 
Proor. By (2) we can transform $\left(a_{0}, \ldots, a_{n}\right)$ to $(1,0, \ldots, 0)$ by an element of $G L_{n+1}(A)$. Therefore we can assume $a_{0}=1, a_{i}=0$ for $i>0$. By [7, Theorem 2] it will suffice to show that $P_{x}$ over $A\left[x, x^{-1}\right]$ extends to $A\left[x^{-1}\right]$. Since $x$ is a unit of $A\left[x, x^{-1}\right], P_{x}$ is also defined by $\left(1+b_{0} x, b_{1}, \ldots, b_{n}\right)$ so it will suffice to show that this row defines a free module over $A[x]$. (A more elementary argument for this is given in $[10,88]$.) Since $1+b_{0} x$ is a unit of $A /\left(b_{1}, \ldots, b_{n}\right)[x]$, we see that $b_{0}^{r} \in \sum_{1}^{n} A b_{i}$ for some $r$ [1, Chapter XII, Proposition 5.2a]. Consider the binomial series

$$
\left(1+b_{0} x\right)^{1 / m}=\sum\left(\begin{array}{c}
1 / m \\
s
\end{array}\right)\left(b_{0} x\right)^{s} .
$$

If $1 / m \in A$, all coefficients lie in $A$. In fact $\left({ }^{2}\right) m^{2 s-1}\left({ }_{s}^{1 / m}\right) \in \mathbf{Z}$. By omitting all terms of degree $>r$ we get a polynomial $f$ with $f^{m} \equiv 1+b_{0} x$ $\bmod \left(b_{1}, \ldots, b_{n}\right)$. By elementary transformations change the unimodular row to $\left(f^{m}, b_{1}, \ldots, b_{n}\right)$. If $m=n$ !, Suslin's theorem shows that $P$ is free. If $n=2$ this is Theorem 8.1 of [10].

COROLLARY 1.7. If $A$ is a commutative ring and $1 / n ! \in A$ then any unimodular row $\left(f_{0}, f_{1}, \ldots, f_{n}\right)$ over $A[x]$ with $f_{0}=1+a x, a \in A$, defines a free module.

Proof. By "dividing" the $f_{i}$ by $f_{0}$ we see that the $f_{i}$ can be replaced by terms of the form $a_{i} t^{r}$ with $a_{i} \in A$. The previous argument then applies.

2. An example. In [5], Loday showed that an orthogonal map $\mathbf{R}^{p+1} \times \mathbf{R}^{q} \rightarrow$ $\mathbf{R}^{q}$ leads to an algebraic map $S^{p} \times S^{q} \rightarrow S^{p+q}$ inducing a homeomorphism $S^{p} \wedge S^{q} \cong S^{p+q}$. I will apply his construction to the orthogonal map $\mathbf{C} \times \mathbf{C}^{n}$ $\rightarrow \mathrm{C}^{n}$ given by $\left(t,\left(z_{1}, \ldots, z_{n}\right)\right) \rightarrow\left(t z_{1}, \ldots, t z_{n}\right)$. Regard $S^{1}$ as the unit sphere of $\mathbf{C}, S^{2 n+1}$ as that of $\mathbf{C}^{n+1}$, and $S^{2 n}$ as that of $\mathbf{R} \times \mathbf{C}^{n}$. After changing the sign of Loday's second real coordinate, his map $S^{1} \times S^{2 n} \rightarrow S^{2 n+1}$ becomes

$$
\begin{aligned}
\left(t,\left(x, z_{1}, \ldots, z_{n}\right)\right) & \\
& \rightarrow\left(1-(1-t)(1-x) / 2,(1-t) z_{1} / 2, \ldots,(1-t) z_{n} / 2\right),
\end{aligned}
$$

where $x \in \mathbf{R}, t, z_{i} \in \mathbf{C}$. It is easy to check directly that this map takes $S^{1} \times S^{2 n}$ to $S^{2 n+1}$ and sends $S^{1} \vee S^{2 n}$ to $(1,0, \ldots, 0)$ where we take $t=1$ and $\left(x, z_{1}, \ldots, z_{n}\right)=(1,0, \ldots, 0)$ as basepoints. To see that $S^{1} \wedge S^{2 n} \rightarrow$ $S^{2 n+1}$ is a homeomorphism, observe that if $\left(a, u_{1}, \ldots, u_{n}\right) \in S^{2 n+1}-\{(1$, $0, \ldots, 0)\}$ then the equations

$$
a=1-(1-t)(1-x) / 2, \quad u_{i}=(1-t) z_{i} / 2
$$

have a unique solution given by $t=(a-1)(1-\bar{a})^{-1}$.

(2) This is easily seen by expanding $\left(1+a_{1} x+a_{2} x^{2}+\ldots\right)^{m}=1+x$ and solving recursively for $a_{s}$. 
If $A$ is any commutative ring with $\frac{1}{2} \in A, x, z_{1}, \ldots, z_{n} \in A$, and $1-x^{2} \in$ $\sum A z_{i}$ then

$$
\left(1-(1-t)(1-x) / 2,(1-t) z_{1} / 2, \ldots,(1-t) z_{n} / 2\right)
$$

is a unimodular row over $A\left[t, t^{-1}\right]$. In fact, the ideal generated by the elements of this row contains $1-(1-t)(1-x) / 2$ and $(1-t)\left(1-x^{2}\right) / 2$. By dividing the second of these by the first we see that the ideal contains $x+1$ and hence also $1-(1-t)=t$.

We now choose $A=\mathbf{C}\left[x_{0}, \ldots, x_{4}\right] /\left(\sum x_{i}^{2}-1\right)$ the ring of complex polynomial functions on $S^{4}$. Consider the unimodular row (*) with $n=2$, $x=x_{0}, z_{1}=x_{1}+i x_{2}$, and $z_{2}=x_{3}+i x_{4}$. This row defines a projective module $P$ of rank 2 over $A\left[t, t^{-1}\right]$.

TheOREM 2.1. $P$ does not extend to $A[x]$ or $A\left[x^{-1}\right]$.

In particular, $P$ is not extended from $A$. Note that $P$ also gives a negative answer to the question at the end of [7].

To prove the theorem, we regard $A\left[t, t^{-1}\right]$ as a ring of functions on $S^{1} \times S^{4}$ by identifying $t$ with the $S^{1}$-coordinate: $S^{1}=\{t \in \mathrm{C}|| t \mid=1\}$. As in [9], $P$ defines a vector bundle $E$ on $S^{1} \times S^{4}$.

\section{LEMMA 2.2. $E$ is nontrivial.}

Proof. The unimodular row (*) defines Loday's map $f: S^{1} \times S^{4} \rightarrow S^{5} \mathrm{C}$ $C^{3}$ and $E$ is therefore the pullback of the bundle $E^{\prime}$ on $S^{5}$ defined by the unimodular row $\left(z_{0}, z_{1}, z_{2}\right)$. It is well known and easy to $\operatorname{see}\left({ }^{3}\right)$ that the associated principal bundle of $E^{\prime}$ is, up to complex conjugation, the canonical bundle $U(2) \rightarrow U(3) \rightarrow S^{5}$. If $E$ is trivial, the pullback of this principal bundle will be trivial and a section of it will give a lifting of $f$ to $g$ : $S^{1} \times S^{4} \rightarrow U(3)$. By [4], $H^{*}(U(3), Z / 2 Z)$ has generators $h_{1} \in H^{1}, h_{2} \in H^{3}$, $h_{3} \in H^{5}$ with $S q^{2} h_{2}=h_{3}$. Since $H^{*}$ is an exterior algebra it is easy to check that $h_{3}$ is the only nonzero element in $H^{5}$. The product formula for Steenrod operations shows that these are all trivial on $S^{1} \times S^{4}$. Therefore $g^{*}\left(h_{3}\right)=$ $S q^{2} g^{*}\left(h_{2}\right)=0$ and so $g^{*}$ is zero in dimension 5. This implies the same for $f^{*}$ which is absurd since $f$ is a map of 5-manifolds of degree 1 .

Suppose now that $P$ has the form $P=A\left[t, t^{-1}\right] \otimes_{A[t]} Q$ where $Q$ is a finitely generated projective $A[t]$-module. Then $Q$ induces the bundle $E$ by the map $A[t] \rightarrow C\left(S^{1} \times S^{4}\right)$ where $C$ denotes the ring of continuous complex functions. Also $Q /(t-1) Q \approx P /(t-1) P$ is free since the unimodular row

( ${ }^{3}$ See D. Husemoller, Fibre bundles, McGraw-Hill, New York,1966, Chapter 7, Theorem 7.71. I would like to thank $C$. Weibel for this reference and for pointing out several misprints. The referee suggested M. Raynaud, Modules projectifs universels, Invent. Math. 6 (1968), 1-26 as an additional reference. Raynaud's methods can be used to extend the results of $\$ 2$ to any field of characteristic $\neq 2$ which contains $\sqrt{-1}$. 
becomes $(1,0, \ldots, 0)$ if $t=1$. The following elementary lemma, with $x=t$ -1 , now leads to the required contradiction.

Lemma 2.3. Let $P$ be a finitely generated projective $R[x]-$ module. Set $Q=P / x P$ and $P^{\prime}=R[x] \otimes_{R} Q$. Let $X$ be a topological space and let $f:$ $R[x] \rightarrow C(X)$ be a ring homomorphism. Then $P$ and $P^{\prime}$ define isomorphic bundles on $X$.

Proof. Let $F: R[x] \rightarrow C(X \times I)$ by taking $R \rightarrow^{f \mid R} C(X) \rightarrow^{\mathrm{pr}^{*}} C(X \times I)$ and sending $x$ to the function $\tau$ with $\tau(a, s)=s[f(x) a]$. If $i_{\nu}: X \rightarrow X \times I$ by $i_{v}(a)=(a, v)$ we see that $f=i_{1}^{*} F$ while $i_{0}^{*} F$ factors as $R[x] \rightarrow R \rightarrow \rightarrow^{f \mid R} C(X)$ and hence also as $R[x] \rightarrow R \rightarrow R[x] \rightarrow^{f} C(X)$. Since $i_{0}$ and $i_{1}$ induce isomorphic bundles [8] the result follows easily.

REMARK. If $P$ and $Q$ are finitely generated projective $R$-modules, write $P \simeq Q$ if there is a finitely generated projective $R[y]$-module $S$ with $S / y S \approx$ $P, S /(y-1) S \approx Q$. We can extend $\approx$ to an equivalence relation. If $P$ is projective over $R[x]$ and $Q=R[x] \otimes_{R}(P / x P)$ then $P \simeq Q$ by taking $S=R[x, y] \otimes_{R[x]} P$ where $R[x] \rightarrow R[x, y]$ by $x \mapsto x y$.

Corollary 2.4. If $f, R \rightarrow C(X)$ and $P \simeq Q$ over $R$ then $P$ and $Q$ induce isomorphic bundles on $X$.

Proof. Consider $R[x] \rightarrow^{j} R \rightarrow^{f} C(X)$ where $j(x)=1$. By Lemma 2.3, $S$ and $P \otimes_{R} R[x]$ induce isomorphic bundles.

REMARK. The construction used to prove Theorem 2.1 does not, of course, apply to $\mathbf{C}\left[t_{1}, t_{1}^{-1}, \ldots, t_{n}, t_{n}^{-1}\right]$. In fact, Loday [5] has shown that any algebraic map $T^{n} \rightarrow S^{n}$ is homotopic to a constant.

The above example can also be used to show that the analogue of [7, Theorem 1] does not hold for Laurent rings.

TheOREM 2.5. Let $A$ and $P$ be as in Theorem 2.1. Then $P_{\mathrm{m}}$ is free over $A_{\mathrm{m}}\left[t, t^{-1}\right]$ for every maximal ideal $\mathrm{m}$ of $A$.

The following special case of the question in [7] therefore remains open: If $A$ is a regular local ring, does every projective $A\left[x, x^{-1}\right]$-module extend to $A[x]$ ? This would, of course, suffice to prove the conjecture in [7].

Theorem 2.5 follows immediately from the definition of $P$ and the following more general result. This depends on Theorem 1.6 and hence on Suslin's as yet unpublished theorem. However, for Theorem 2.5 we only need the case $n=2$ which follows from [11, Theorem 2.1].

LEMMA 2.6. Let $A$ be a commutative local ring and let $\left(f_{0}, f_{1}, \ldots, f_{n}\right)$ be a unimodular row over $A\left[t, t^{-1}\right]$ which defines a projective module $P$. Assume 
(1) Each $f_{i}$ has the form $f_{i}=a_{i}+b_{i} t, a_{i}, b_{i} \in A$,

(2) $1 / n ! \in A$.

Then $P$ is free.

Proof. Since $A$ is local, $P /(t-1) P$ is free. It is defined by $\left(f_{0}(1), \ldots, f_{n}(1)\right)$ so we can transform this row to $(1,0, \ldots, 0)$ by an element of $G L_{n+1}(A)$. By using the same transformation on the original row we can assume $f_{0}=1+(t-1) b$ and $f_{i}=(t-1) b_{i}$ for $i>0$. Let $\mathfrak{p}$ be a prime ideal of $A$ containing $b_{1}, \ldots, b_{n}$ and let $K \supset A / \mathfrak{p}$ be a field. Extend $A \rightarrow K$ to $A\left[t, t^{-1}\right] \rightarrow K$ by $t \mapsto \tau \neq 0$. If $b \mapsto \beta \in K$ then $f_{0} \mapsto 1+(\tau-1) \beta$ which must be nonzero by the unimodularity. But if $\beta \neq 0$ or 1 we can set $\tau=1-\beta^{-1}$. Therefore $\beta(\beta-1)=0$ so $b(b-1) \in \mathfrak{p}$. It follows that $b^{m}(b$ $-1)^{m} \in \sum_{1}^{n} A b_{i}$ for some $m$. Since $A$ is local, one of $b$ or $b-1$ is a unit. Therefore either $b^{m}$ or $(b-1)^{m}$ lies in $\Sigma A b_{i}$. In the first case, $\left(f_{0}, f_{1}, \ldots, f_{n}\right)$ is unimodular over $A[t]$ and gives an extension of $P$ to this ring. Theorem 1.6 then shows that $P$ is free. In the second case, the row is equivalent to

$$
\begin{aligned}
& \left(t^{-1}+\left(1-t^{-1}\right) b,\left(1-t^{-1}\right) b_{1}, \ldots\right) \\
& =\left(1+\left(1-t^{-1}\right)(b-1),\left(1-t^{-1}\right) b_{1}, \ldots\right)
\end{aligned}
$$

which is unimodular over $A\left[t^{-1}\right]$ and the same argument applies.

3. Schemes. If $\mathbf{A}^{1}=\operatorname{Spec} Z \mathbf{Z}[x]$, then $\operatorname{Spec} A[x]=\operatorname{Spec} A \times \mathbf{A}^{1}$. This suggests trying to extend [7, Theorem 1] to vector bundles on schemes of the form $X \times \mathbf{A}^{1}$. An extended bundle here means one of the form $p^{*} \mathcal{F}$ where $p$ : $X \times \mathbf{A}^{1} \rightarrow X$ is the projection and $\mathscr{F}$ is a bundle on $X$. I will show here that this generalization is false $\left.{ }^{4}\right)$.

THEOREM 3.1. Let $R$ be a regular local ring of dimension 2 with maximal ideal $\mathfrak{m}=\left(a_{1}, a_{2}\right)$. Let $X=\operatorname{Spec} R-\{\mathfrak{m}\}=X_{1} \cup X_{2}$ where $X_{i}=\operatorname{Spec} R_{a_{i}}$. Then there is a vector bundle $\mathscr{F}$ of rank 2 on $X \times \mathbf{A}^{1}$ which is not extended but whose restrictions to $X_{i} \times \mathbf{A}^{1}, i=1,2$ are free.

Proof. Let $k=R / \mathfrak{m}=R[x] /\left(a_{1}, a_{2}, x\right)$ and consider the exact sequence

$$
0 \rightarrow M \rightarrow R[x]_{a_{1}, a_{2}, x}^{3} R[x] \rightarrow k \rightarrow 0 .
$$

Let $\tilde{M}$ be the sheaf on Spec $R[x]$ defined by $M$ and let $\mathscr{F}$ be the restriction of $\tilde{M}$ to $X \times A^{1}$. It is trivial that $\mathscr{F}$ is free on $X_{i} \times A^{1}=\operatorname{Spec} R_{a_{i}}[x]$.

Now collapsing (*) modulo $x$ gives

$$
0 \rightarrow M / x M \rightarrow R^{3} \underset{a_{1}, a_{2}, 0}{\rightarrow} R \rightarrow k \rightarrow 0
$$

(4) The referee informs me that this is well known for $X=P^{1}$. Theorem 3.1 can also be derived easily from this. 
which is exact at all points of Spec $R$ except $m$. This sequence gives us a map $f: M / x M \rightarrow R^{2}$ since the Koszul resolution

$$
0 \rightarrow R \rightarrow R_{a_{1}, a_{2}}^{2} R \rightarrow k \rightarrow 0
$$

is exact, and $f$ is clearly an isomorphism except at $\mathfrak{m}$. Since $(M / x M) \sim \mid X=$ $\mathscr{F} / x \mathcal{F}$, we see that $\mathscr{F} / x \mathscr{F} \approx \theta_{X}^{2}$. Therefore, if $\mathcal{F}$ is extended then $\mathscr{F} \approx$ $\left(\theta_{X \times A^{\prime}}\right)^{2}$. By Lemma 3.2 below we would have $M \approx \Gamma\left(X \times A^{1}, \mathscr{F}\right) \approx \Gamma(X \times$ $\left.\mathbf{A}^{\mathbf{1}}, \theta\right)^{2} \approx R[x]^{2}$ but this is impossible because $k$ has projective dimension 3 over $R[x]$.

To complete the proof we need the following result which was pointed out to me by Murthy.

LEMMA 3.2 (MURTHY). Let $X$ be a normal, locally noetherian scheme. Let $U$ be an open set of $X$ such that $X-U$ has codimension $>2$ in $X$. Let $\mathscr{F}$ and $\mathcal{G}$ be coherent sheaves on $X$ such that locally on $X$ one can find exact sequences of the form

$$
0 \rightarrow \mathcal{G} \rightarrow \theta_{X}^{m} \rightarrow \theta_{X}^{n}
$$

with $m, n<\infty$. Then

$$
\operatorname{Hom}_{x}(\mathscr{F}, \mathcal{G}) \stackrel{\approx}{\rightarrow} \operatorname{Hom}_{U}(\mathscr{F}|U, \mathcal{G}| U) .
$$

Proof. The question is clearly local on $X$. Therefore we can assume $0 \rightarrow \mathcal{G} \rightarrow \mathcal{O}^{m} \rightarrow \mathcal{O}^{n}$. This gives

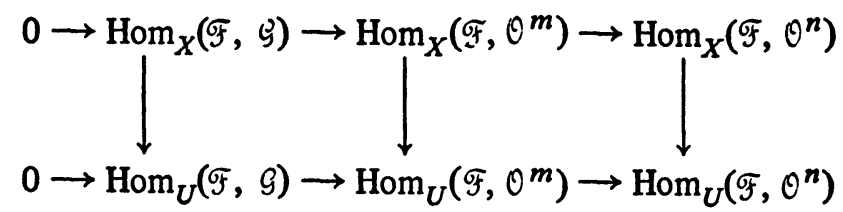

which shows that it is sufficient to consider the case $\mathcal{G}=\mathcal{O}$. Similarly, using a local resolution $\theta^{r} \rightarrow \theta^{s} \rightarrow \mathscr{F} \rightarrow 0$ we can reduce to the case $\mathscr{F}=\theta$. Thus it is sufficient to show that $\Gamma(X, \theta) \rightarrow \approx \Gamma(U, \theta)$ locally on $X$ but this follows from the fact that $R=\cap R_{\mathfrak{p}}$ over prime ideals of height 1 if $R$ is a normal noetherian domain.

REMARK. The hypothesis on $\mathcal{G}$ is clearly satisfied if $\mathcal{G}=\operatorname{Hom}(\mathcal{H}, \theta)$ with $\mathcal{H}$ coherent. We need only take a local resolution of $\mathcal{X}$. In particular, it is satisfied if $\mathcal{G}$ is reflexive.

In a sense it is rather unfortunate that [7, Theorem 1] does not hold for schemes since this would have the following remarkable consequence: If $R$ is a normal noetherian domain then all projective $R[x]$-modules are extended. I do not know if this is true. By [7, Theorem 1] it would suffice to do the case where $R$ is local. Suppose it is true for rings of smaller Krull dimension. If $P$ is projective over $R[x]$ then, using the notation of the proof of Theorem 3.1, 
we let $\mathscr{F}=\tilde{P} \mid X \times \mathbf{A}^{1}$. This is locally extended by the induction hypothesis. If it is extended, then $\mathscr{F} \approx 0_{X \times A^{\prime}}^{n}$ so $P \approx R[x]^{n}$ by Lemma 3.2.

It is not even known if this result holds stably [2, $\$ 2$, p. 15]. It would be enough to do this for local rings $R$ by Lemma 1.5 .

REMARK. It is not hard to check that the bundle $\mathscr{F}$ used in the proof of Theorem 3.1 is obtained by glueing free sheaves over $X_{1} \times \mathrm{A}^{1}$ and $X_{2} \times \mathrm{A}^{1}$ by an automorphism of the form

$$
\left[\begin{array}{cc}
1 & \frac{x}{a_{1} a_{2}} \\
0 & 1
\end{array}\right] \in G L_{2}\left(R_{a_{1} a_{2}}[x]\right) .
$$

This shows that the proof in [7] does not apply because $1 / a_{1} a_{2} \notin R_{a_{1}}+R_{a_{2}}$ in contrast to the case where $R a_{1}+R a_{2}=R$. If $\mathscr{F}$ comes from a projective module $P$ over $R[x]$, an obstruction of this simple type does not arise since $R[[x]] \otimes_{R[x]} P$ is free. In fact, Murthy [6] has shown that all projective $R[x]$-modules are free if $R$ is a 2-dimensional regular local ring. He has recently extended this argument to show that all projective $R\left[x_{1}, \ldots, x_{n}\right]-$ modules are extended if $R$ is regular of dimension 2.

\section{REFERENCES}

1. H. Bass, Algebraic K-theory, Benjamin, New York, 1968. MR 40 \#2736.

2. Some problems in "classical" algebraic $K$-theory, Algebraic $K$-Theory, II (Proc. Conf., Seattle Res. Center, Battelle Mem. Inst., 1972), Lecture Notes in Math., vol. 342, Springer-Verlag, Berlin and New York, 1973, pp. 3-73. MR 48 \#3656b.

3. Libération des modules projectifs sur certains anneaux de polynômes, Séminaire Bourbaki 1973/1974, Exposé no. 448, Lecture Notes in Math., vol. 431, Springer-Verlag, Berlin and New York, 1975. pp. 228-254. MR 51 \# 18.

4. A. Borel and J.-P. Serre, Groupes de Lie et puissances réduites de Steenrod, Amer. J. Math. 75 (1953), 409-448. MR 15, 338.

5. J.-L. Loday, Applications algébriques du tore dans la sphère et de $S^{p} \times S^{q}$ dans $S^{p+q}$, Algebraic $K$-Theory, II (Proc. Conf., Seattle Res. Center, Battelle Memorial Inst., 1972), Lecture Notes in Math., vol. 342, Springer-Verlag, Berlin and New York, 1973, pp. 79-91. MR 51 \#4276.

6. M. P. Murthy, Projective A[X]-modules, J. London Math. Soc. 41 (1966), 453-456. MR 34 \#188.

7. D. Quillen, Projective modules over polynomial rings, Invent. Math. 36 (1976), 167-171.

8. N. Steenrod, The topology of fibre bundles, Princeton Univ. Press, Princeton, N. J., 1951. MR 12, 522.

9. R. G. Swan, Vector bundles and projective modules, Trans. Amer. Math. Soc. 105 (1962), 264-277. MR 26 \#785.

10. - Serre's problem, Conf. on Commutative Algebra-1975 (A. Geramita, Editor), Queen's Papers in Pure and Appl. Math., no. 42, Queen's Univ., Kingston, Ontario, Canada.

11. R. G. Swan and J. Towber, A class of projective modules which are nearly free, J. Algebra 36 (1975), 427-434.

Department of Mathematics, Untversity of Chicago, Chicago, Illinois 60637 